\section{TUBAL PREGNANCY.}

By Henry Hancox, M.D., Wolverhampton.

THE subject of the following case was Mrs. D., the wife of a miner, residing at Bilston. She was the mother of one child which survived its birth only a few hours; the catamenia had been regular up to the period of her becoming pregnant with the present child. She is 23 years of age, and has always enjoyed good health. On September 24th, she began to complain of pain on the right side of the abdomen, accompanied with a sinking sensation and faintness, which she attributed to an injury she had received on September 4 th, in defending herself against a man who attempted to commit a rape upon her. A dose of opium was administered, which gave her relief: and she continued to go about and discharge her domestic duties, until November 17th, when the pain in the right side of the abdomen again returned, and was again relieved by the same medicine.

November 21st. She became much worse, the pain being of a more violent character, accompanied with great nervous depression. On examination per vaginam, the parts were found very dry; the os uteri was very high up; tilted forward under the pubis; no signs of labour could be detected. She continued to get worse; the symptoms becoming more alarm. ing; the pulse was feeble and quick; colliquative sweats bedewed the body; the eyes were sunken, and the countenance was pallid, and presented an anxious expression; she lingered in this state till the 23rd, when death ended her sufferings.

On the 25 th, a post mortem examination was made by my brother, Mr. W. M. Hancox, in the presence of Dr. Fraser, Mr. Gutteridge, Mr. Larkin, and myself, when the following appearances were observed :-On laying open the cavity of the abdomen, a female child, together with the placenta, was found in the cavity of the abdomen; the child was supposed by all present to be at least eight months and a half. It had been well nourished; and, judging from appearance, it must have been alive at the time of the poor woman's death. The placenta was well formed, and did not differ in any respects from an uterine placenta, but presented a natural and healthy appearance. The cavity of the abdomen contained at least four or five quarts of effused and coagulated blood. On examining the uterus minutely, to find the place where rupture had taken place, we found a large opening three or four inches in length, in what afterwards proved to be the right Fallopian tube, the cavity of which was dilated to the size of a naturally impregnated womb at the ninth month, and presented all the appearances of the gravid uterus at the same period of gestation. The walls of the cavity were very thin, and attenuated to the breadth of three fingers at its superior edge, the place where rupture had taken place; while below this spot, the walls were very much thickened, like the substance of the heart. The communication between the Fallopian tube and the uterus was perfectly occluded. The body of the uterus was found in its natural state; it was not enlarged. On laying it open, the internal surface was in a perfectly healthy condition, and an opening into the Fallopian tube of the left side was quite apparent, but no trace of a similar communication into the right tube was discovered. The ovaria presented no unusual appearances; the Graafianfvesicles were distinctly visible, especially in the right ovaria.

REMARKS. In recording the above interesting case, I am convinced in my own mind, that it will not fail to be interesting to many readers of the JOURNAL, not only from the circum stance attending the death of the poor woman, but from the length of time she carried the child in the Fallopian tube without experiencing any pain or inconvenience till within a few weeks of her death; the Fallopian tube admitting of so much dilatation as to be able to contain so well nourished and large a child (eight months and a half) and a placenta, in every way as large and as thick as an uterine placenta, renders it one of the most remarkable cases I am acquainted with on record. Dr. Burns (Principles of Midwifery, tenth edition) mentions a case occurring in the practice of a Mr. Moore, in which the woman carried the foetus to the sixth month, the uterus being enlarged to the size of a fist. The uterus in this instance was no larger than an unimpregnated womb. This circumstance does not accord with the experience of Dr. Burns, who states, "it generally enlarges considerably". Dr. Hunter, he says, "had a preparation of tubal pregnancy in which the uterus was found enlarged to double its natural size." It is a matter of surprise that rupture did not take place immediately, or soon after the injuries she received six or seven weeks 1022 previous to her death; but she seems to have completely recovered from the injury and shock which the system must necessarily have received, as to be able to go about and discharge her duties.

\section{筑otes of aldtsominat 进ectures} ox

\section{CERTAIN POINTS CONNECTED WITH DIABETES.}

DELIVERED BEFORE THE MEDICAI SOCIETY OF LONDON.

By FREDERICK WM. PAVY, M.D., F.R.C.P., Lecturer on Physiology at Guy's Hospital.

LECTURE III.

[Delivered December 7th, 1859.]

THE views promulgated by $M$. Bernard, as to the functions of the liver, have met with some amount of opposition on the Continent. M. Figuier admits the presence of sugar in the animal body; he believes, however, that it has not been formed there, but that it has been derived from the vegetable kingdom and stored up. To explain the absence of sugar in the blood of the portal vein, he supposes that there is something in that fluid which masks its presence. In 1850, M. Schmidt published some views which referred sugar to a change taking place in the circulatory system, not in the liver. Again, M. Sanson has promulgated a theory in which he follows $M$. Figuier to some extent; but he says that a material closely allied to the glucogenic matter is found in all the tissues as well as in the liver. These objections, however, to M. Bernard's theory are quite destitute of validity, being entirely hypothetical.

In the experiment recorded at the end of the second lecture, there was sufficient evidence to show that the liver has not a sugar-forming function during life. Since, then, the liver does not possess such a function, and since the blood from this organ, examined during life, gives no trace of sugar, it was necessary to modify the opinion which we have been led by $\mathbf{M}$. Bernard to entertain; and we have now to ask, What becomes of the amyloid substance? And here there is a difficulty: for it is often more easy to overturn a theory than to substitute a satisfactory one for it. Dr. Pavy, however, was engaged in the investigation of the question; and he believed he had some landmarks in the inquiry which seemed likely to lead him to the determination of a close relation between the amy. loid substance and fat. The amyloid substance, as was shown in some experiments referred to in the second lecture, is notably increased in quantity by the use of food containing sugar; we know also that fat may be formed from saccharine matter. If an animal be fed with material containing starch or sugar, it becomes fat; and in the West India islands, it is a matter of common observation that the natives become fat during the sugar season, from their habit of sucking the canes. The amyloid substance seems to hold an intermediate position between sugar and fat. Further observations on this subject, however, Dr. Pavy would defer until his investigations were more complete.

An unnatural conversion of the amyloid substance into sugar leads to diabetes. This disease depends on the presence of sugar in the blood: if this substance be accumulated in the blood in sufficient quantity, it will appear in the urine. This is true also of grape-sugar, if injected into a vein ; cane-sugar, introduced into the system in this way, appears in the urine as cane-sugar; but if introduced by the alimentary canal in sufficient quantity to cause diabetes, it is transformed into grapesugar. Dr. Pavy had observed this in experiments on dogs. Starting from this point, it might now be asked, How does the sugar enter the blood?

According to the former doctrine, there were two ways in which sugar might enter the circulatory system. M. Bernard taught that sugar was formed in the liver, and conveyed thence to the lungs. If more was formed than could be eliminated by the lungs, it would be accumulated in the blood; and the same result would arise if the power of the lungs to destroy the ordinary amount of sugar were defective.

But the hypothesis that sugar undergoes combustion in the lungs, is negatived by the fact that, whenever it is found in the right side of the heart, it is also found in the arterial system, 
having been carried through the lungs without change. Some experiments made by Dr. Pavy established this point : in all, he had found as much sugar in the blood of the arterial sys. tem as in that of the veins.

It is very probable that, in diabetes, the liver is the seat of the formation of sugar. Under what circumstances may this occur? Dr. Pavy thought that, if from any cause the amyloid substance in the liver escaped into the blood, it was at once transformed into sugar; and again, that a transformation analogous to that which occurs after death might take place in the liver during life. He had ascertained by experiment that the introduction of the amyloid substance into the circulation would produce diabetes. He had injected some into the venous sys. tem of a rabbit; and had found sugar in the urine withdrawn an hour afterwards, none having been present in the urine passed at the time of the experiment. Blood, indeed, acts as an exceedingly energetic ferment on the amyloid substance, whether within or out of the body. Dr. Pavy thought that the presence of saccharine urine in coma, hooping-cough, pneumonia, and other disorders attended by great and sudden congestion, was to be explained by the pressure on the cells containing the amyloid substance causing them to discharge their contents into the circulatory system. Some time ago, he muffled a dog's nose, so as to produce a comatose state; in an hour, the animal's urine was highly saccharine. He hal also covered the head of an animal with a bladder, fitted with a tube and plug, by which the supply of air could be regulated, so as to produce an interference with the circulation short of death. The urine passed immediately before the experiment gave no indication of sugar; but that withdrawn afterwards contained this substance. The occurrence of sugar in the urine in congestive states is a well known fact.

If it is possible for the amyloid substance under certain circumstances to enter the circulation, why does it not always escape from the liver-cells into the blood? Dr. Pavy observed, that this was to be explained on physical principles. The amyloid substance is incapable, under ordinary circumstances, of passing by endosmose through animal membranes; and in this respect it resembles albumen, but differs greatly from sugar, the diffusive power of which is considerable. It has long been known that sugar or urea passes rapidly through membranes; and that albumen does not readily pass by endosmose. If albumen were as diffusible as urea, it would be constantly eliminated, and a vast portion of the material intended for the nutrition of the body would be lost. But if the renal veins be obstructed so as to produce congestion, albumen appears in the urine, because of the distension of the blood-vessels, and of the pressure consequently exerted. The passage of albumen through a membrane may also be artificially produced by ex. periment out of the body. If a piece of animal membrane be tied across the mouth of a tube, and an albuminous liquid be placed in it, and the tube immersed in water, the albumen will not pass through; and the same is the case with amyloid sub. stance. But if a long tube is filled with albuminous liquid to the height of about three feet, then the pressure is sufficient to cause the transudation of the albumen. For the amyloid substance, a pressure of six feet is required to cause it to appear in the outer vessel at the end of forty-eight hours. This is an interesting fact. When the circulation in the liver is not congested, and the cells containing the amyloid substance are not unduly pressed on, then the blood does not carry away any of the substance.

We have now to examine the circumstances under which sugar appears unnaturally in the liver, and is removed by the blood. And here M. Bernard's observations on the effects of division of the spinal cord and on the influence of cold, overturn his own theory. The condition of the bile also affords an important contradiction to the theory of the Parisian physiologist. If sugar really were formed in the liver, it would be found in the bile: but no traces of sugar can be detected in this secretion obtained from the animal during life or imme. diately after death. Bernard, indeed, says that, if an animal be killed and placed aside for a day, the bile in the gall-bladder will be found to contain an abundance of sugar: this, however, arises from a change occurring after death.

It occurred to Dr. Pavy, that by keeping up the circulation by means of artificial respiration in an animal killed by pithing, diabetes would be established; and in this experiment he has succeeded. It is necessary, however, that the life of the animal be completely destroyed: and it is further observed, that the medulla oblongata appears more intimately connected with the formation of sugar than any other part of the nervous system. If the spinal cord is divided immediately below the medulla, respiration is of course arrested, but saccharine urine is not produced; but if the medulla oblongata is divided, then sugar appears. Again, if the cerebral peduncles be divided, there is no production of sugar. The medulla oblongata, therefore, seems to preside over the life of the liver.

Irritation, or rather puncture, of the floor of the fourth ventricle has been found to produce diabetes. Bernard explains. this by supposing that the liver has been thereby stimulated to the production of an excessive quantity of sugar. But it is more likely that some power is taken from the liver by the injury; that it is thereby placed in the same condition as after death.

The channel of communication between the medulla and the liver had been sought to be ascertained by Dr. Pavy. It is not the pneumogastric nerves, nor the spinal cord; for both these have been repeatedly divided, both singly and together, without. producing diabetes. The only remaining source, then, is the sympathetic: and the experiments by which Dr. Pavy has sought to determine the function of this part of the nervous system as a communication between the medulla and the liver, are contained in the recently published volume of Guy's Hospital Reports.

After observing the results of dividing the spinal cord, medulla, cerebral peduncles, and pneumogastric nerve, Dr. Pavy decapitated an animal, and kept up artificial respiration, care having been taken to prevent much loss of blood. In threequarters of an hour, the urine in the bladder was found to be strongly saccharine. It being thus sufficiently plain that the sympathetic was the medium of communication, Dr. Pavy proceeded to examine as to what part of the nerve had this function. There are some large filaments of this nerve lying close beside the vertebral artery; these were accordingly divided on each side. In the first experiment of this kind, Dr. Pavy found the urine saccharine in twenty minutes. But he also found that, if these filaments were divided higher up, no diabetes was produced, unless the carotids were also tied. Saccharine urine is produced in a marked degree in cases where the superior cervical ganglion is punctured or removed. In one such experiment, the urine at the end of an hour yielded 20.5 grains of sugar in the ounce; an hour afterwards, the amount had fallen to 11.4 grains; and next day there was no appearance of sugar. As the result of several experiments, Dr. Pavy found that division of the splanchnic nerves causes diabetes, but not uniformly; the reason of this irregularity, however, he could not ascertain.

It is important to observe, that the effects of lesion of the sympathetic in producing saccharine urine may be prevented by injecting an alkali, as carbonate of soda, into the circulation. The injection of two hundred grains of carbonate of soda is sufficient for this purpose; one hundred grains fail to produce the effect. Dr. Pavy had tried to make practical application of this fact in the hospital wards, by administering citrate of potash to aiabetic patients. But he found that, although under ordinary circumstances the injection of such salts renders the urine alkaline, it has no such effect in diabetic urine, which remains strongly acid. In one case in which citrate of potash was given, the quantity of urine and the proportion of sugar were both reduced; but in other cases this did not occur.

To explain the action of alkalies in preventing transformation of the amyloid substance into sugar, Dr. Pavy supposed that this substance may be normally combined with an alkali, which prevents transformation; while, if the alkali be removed by something for which it has a more powerful affinity, the amyloid substance is left at liberty to undergo transformation.

Having advanced this far in our knowledge of the physiology of the formation of sugar in the body, it is an important object to discover what will prevent the transformation of the amyloid substance during life; and it is to be hoped that it will not be long before our positive knowledge on this matter will be greater than it is at present.

Uniform of ARMy Medical Officers. The Queen has been pleased to approve a new code of regulations for the dress of the officers of the Army Medical Department. The tunic in future to be worn is scarlet, single-breasted, with black facings edged with white cloth, the distinctions of rank to be regulated by the badges and lace, according to the relative ranks in the army. 